\title{
Safety and Immunogenicity of Different Immunization Regimens of CVD 103-HgR Live Oral Cholera Vaccine in Soldiers and Civilians in Thailand
}

\author{
Pisit Su-Arehawaratana, Preecha Singharaj, \\ David N. Taylor, Charles Hoge, ${ }^{*}$ Andrew Trofa, \\ Krit Kuvanont, Sricharoen Migasena, \\ Punnee Pitisuttitham, Yu Leung Lim, \\ Genevieve Losonsky, James B. Kaper, \\ Steven S. Wasserman, Stanley Cryz, \\ Peter Echeverria, and Myron M. Levine
}

\author{
Medical Corps, Royal Thai Army, Armed Forces Research Institute of \\ Medical Sciences, and Vaccine Trial Centre, Faculty of Tropical \\ Medicine, Mahidol University, Bangkok. Thailand; Department of \\ Enteric Infections, Walter Reed Army Institute of Research, Washington, \\ $D C$; Center for Vaccine Development, University of Maryland School of \\ Medicine, Baltimore, Maryland; Swiss Serum and Vaccine Institute,
} Bern, Switzerland

\begin{abstract}
Attenuated Vibrio cholerae oral vaccine CVD 103-HgR was well tolerated by 324 Thai soldiers and civilians. Most received a single $5 \times 10^{8}$ cfu dose, while 40 each received one or two $5 \times 10^{9}$ cfu doses. Vibriocidal antibody (the best correlate of immunity) seroconversion was lower in soldiers than civilians $(P<.001)$. Increasing the vaccine dose to $5 \times 10^{9} \mathrm{cfu}$ raised the geometric mean titer $(P<.001)$. A second $5 \times 10^{9}$ cfu dose one week later did not notably increase seroconversions. Likelihood of seroconversion was inversely correlated with baseline vibriocidal titer $(P$ $<.001$ ). CVD 103-HgR caused seroconversion in most subjects with baseline titers $\leqslant 1: 40$, including $100 \%$ of civilians after one $5 \times 10^{8} \mathrm{cfu}$ dose, $79 \%$ of soldiers after one $5 \times 10^{9} \mathrm{cfu}$ dose, and $45 \%$ of soldiers after one $5 \times 10^{8} \mathrm{cfu}$ dose. In persons with elevated baseline titers, vibriocidal antibody seroconversion is not a sensitive measure of whether vaccine has boosted intestinal immunity; for such subjects, other measurements must be used. Study regimens in endemic areas should use a single $5 \times 10^{9} \mathrm{cfu}$ dose.
\end{abstract}

Cholera remains an important public health problem in less-developed countries, spreading readily where sanitation is compromised and often appearing in explosive epidemics. The World Health Organization has targeted the development of an improved cholera vaccine as a priority $[1,2]$ because the parenteral inactivated whole cell vaccine, which provides only limited, short-lived protection, can play no practical role in cholera control [3]. An ideal new cholera vaccine would be well tolerated and rapidly stimulate a high level of long-term protection among all age groups after administration of just one oral dose. Such a vaccine would constitute a welcome addition to the public health intervention measures available to control epidemic and endemic cholera.

An important advance in immunization against cholera was documented several years ago in a field trial in Bangla-

Received 6 November 1991; revised 14 January 1992

The clinical protocol followed the guidelines of the Department of Health and Human Services and was reviewed by ethical committees at the University of Maryland at Baltimore, Mahidol University, and the US Department of the Army. The studies were explained in detail, and written informed consent was obtained.

Grant support: National Institutes of Health (AI-62528), Swiss Serum and Vaccine Institute, and US Agency for International Development.

Reprints or correspondence: Dr. M. M. Levine, Center for Vaccine Development, University of Maryland School of Medicine, $10 \mathrm{~S}$. Pine St.. Baltimore, MD 21201 .

* Present affiliation: Department of Bacteriology. Armed Forces Research Institute of Medical Sciences, Bangkok, Thailand.

The Journal of Infectious Diseases 1992;165:1042-8 (C) 1992 by The University of Chicago. All rights reserved. 0022-1899/92/6506-0009\$01.00 desh when two related inactivated oral cholera vaccines (one consisting of inactivated Vibrio cholerae $\mathrm{Ol}$ organisms and the other of inactivated organisms plus the B subunit of cholera toxin) were each shown to confer $50 \%$ protection for 3 years [4]. That experience illustrates that oral vaccines can elicit relatively long-lived protection against cholera. However, the field trial in Bangladesh also exposed notable deficiencies of those oral inactivated vaccines: Multiple, spaced doses were required to elicit protection, young children (the population with the highest incidence of cholera in that area) were least protected, and despite administration of three spaced doses, the level of efficacy was only $50 \%-52 \%$ [4].

With attenuated strains of $V$. cholerae $\mathrm{Ol}$ as live oral vaccines, it may be possible to overcome the drawbacks of the oral inactivated vaccine and to protect satisfactorily after just one dose of vaccine. CVD $103-\mathrm{HgR}$ is an attenuated strain of $V$. cholerae $\mathrm{Ol}$ derived from wild-type classical Inaba strain $569 \mathrm{~B}$ by deleting the genes that encode the A subunit of cholera toxin [5] and by inserting a gene encoding resistance to $\mathrm{Hg}^{2+}$ into the $h l y \mathrm{~A}$ locus of the chromosome [6, 7]. CVD $103-\mathrm{HgR}$ has been given to $\sim 1500$ adults and children in phase 1 and 2 clinical studies in industrialized and in less-developed countries; the vaccine was well tolerated with no adverse reactions attributable to the vaccine over the background frequency of reaction among placebo recipients.

Since vibriocidal antibody is currently recognized as the best correlate of protection and of the successful stimulation of antibacterial immunity (whether infection- or vaccinederived) $[8-11]$, measurement of the serum vibriocidal antibody response has been used as the main gauge of the 
immunogenicity of CVD 103-HgR [6, 12, 13]. The immunogenicity of this vaccine has been consistently impressive in adults in industrialized countries $[6,7,12]$. For example, $92 \%$ of US adults who ingested a single oral dose containing $5 \times 10^{8} \mathrm{cfu}$ of CVD 103-HgR showed fourfold or greater rises in serum vibriocidal antibody, $49 \%$ reached titers $\geqslant 1: 2560$, and the geometric mean titer (GMT) was 1596 (unpublished data). Similar results were observed in Swiss adults who received a single $5 \times 10^{8} \mathrm{cfu}$ oral dose of CVD $103-\mathrm{HgR}$ [8]. Titers of $\geqslant 1: 2560$ arbitrarily have been considered high and previously have been used as a useful parameter in evaluations of oral cholera vaccines $[6,8,12-15]$.

On the basis of encouraging results obtained in adults in industrialized countries, a preliminary study of the safety and immunogenicity of CVD $103-\mathrm{HgR}$ was done in young adults under containment in the Research Ward of Mahidol University's Vaccine Trial Centre [13]; 24 subjects were randomized to receive a single $5 \times 10^{8} \mathrm{cfu}$ oral dose of CVD 103HgR or placebo. In this study the vibriocidal antibody responses surpassed those recorded for North Americans. Following that initial inpatient volunteer study in Thailand, we carried out a series of additional phase 2 field studies in Thailand to assess the safety and immunogenicity of CVD $103-\mathrm{HgR}$. Here we report results of a series of studies in Thai soldiers and civilians that compares different dosages, immunization schedules, and lots of this oral vaccine.

\section{Materials and Methods}

\section{Vaccine and Placebo Preparations}

The vaccine formulation consisted of two packets: One contained lyophilized vaccine $\left(5 \times 10^{8}\right.$ or $\left.10^{9} \mathrm{cfu}\right)$ with aspartame, while the other contained buffer $\left(2.5 \mathrm{~g}\right.$ of $\mathrm{NaHCO}_{3}$ and $1.65 \mathrm{~g}$ of ascorbic acid). A packet of vaccine and a packet of buffer were mixed in a cup containing $100 \mathrm{ml}$ of water and the suspension was given orally to healthy subjects. In some studies controls received a placebo preparation consisting of a packet containing $5 \times 10^{8}$ inactivated, lyophilized Escherichia coli K12. The placebo powder appeared identical to the vaccine before and after mixing with buffer.

\section{Subjects}

Volunteers were soldiers in the Royal Thai Army or civilians 18-26 years of age. None of the Royal Thai Army soldiers had received parenteral inactivated whole cell cholera vaccine previously.

\section{Study Design}

Four studies were done during February 1988-June 1991 in Thai soldiers or civilians.

Study 1, February 1988. A total of 206 Thai soldiers were randomly allocated to receive one $5 \times 10^{8}$ cfu dose of CVD
Table 1. Design of study 4 to evaluate tolerance and immunogenicity of different immunization regimens of live oral cholera vaccine CVD 103-HgR in Thai soldiers.

\begin{tabular}{lcc}
\hline & \multicolumn{2}{c}{ Preparation* given on day } \\
\cline { 2 - 3 } Group & 0 & 7 \\
\hline A & $5 \times 10^{9}$ & $5 \times 10^{9}$ \\
B & Placebo & $5 \times 10^{9}$ \\
C & $5 \times 10^{9}$ & Placebo \\
D & $5 \times 10^{8}$ & Placebo \\
E & Placebo & $5 \times 10^{8}$ \\
F & $5 \times 10^{9}$ & $5 \times 10^{9}$ \\
\hline$*$ & Colony-forming units of attenuated vaccine strain CVD 103- $\mathrm{HgR}$.
\end{tabular}

103-HgR oral vaccine or placebo and were examined daily for 7 days for adverse reactions. Serum for antibody measurement was collected before and 9,21 , and 28 days after vaccination.

Study 2, November 1988. Forty Thai soldiers were vaccinated orally with one $5 \times 10^{8} \mathrm{cfu}$ dose of vaccine. They were randomized to receive doses from either the identical lot of vaccine that was used in study 1 or another lot. Subjects were monitored for 7 days for adverse reactions, and sera were collected before and 9 and 28 days after vaccination for antibody measurement.

Study 3, March 1989. A group of 33 soldiers and a group of 30 civilians each received a single $5 \times 10^{8} \mathrm{cfu}$ oral dose from the identical lot of vaccine given identically under supervision of the same clinical investigator. Subjects were followed for 7 days for adverse reactions, and sera were obtained before and 9 and 28 days after vaccination.

Study 4, June 1991. The unexpectedly low overall rates of vibriocidal antibody seroconversion of soldiers given a single 5 $\times 10^{8}$ cfu dose of CVD 103- $\mathrm{HgR}$ led us to design a study to answer several practical questions: Whether a single $5 \times 10^{9} \mathrm{cfu}$ dose would be significantly more immunogenic than a $5 \times 10^{8}$ cfu dose; whether a second $5 \times 10^{9} \mathrm{cfu}$ dose of vaccine 1 week after the first dose would enhance immunogenicity; and whether the same regimen of vaccine given to several different groups of subjects would result in immune responses that were consistent. We attempted to answer these questions in a randomized, multigroup (20 subjects/group), double-blind, crossover study (table 1).

The 120 orally vaccinated subjects were followed for 14 consecutive days to detect adverse reactions. Sera were collected before and 7, 14,21, and 28 days after the first dose of vaccine or placebo.

\section{Definitions of Adverse Reactions}

Diarrhea was defined as the passage of at least four loose stools within a $24-\mathrm{h}$ period. One or more episodes of emesis was considered vomiting.

\section{Serologic Methods}

Inaba vibriocidal antibody was measured by the microtiter method [16]; fourfold or greater rises in titer were considered 
significant (seroconversion). IgG cholera antitoxin was measured by ELISA in serum specimens diluted 1:50 as previously described [17]; when the net optical density (OD) of the prevaccination specimen was $\geqslant 1.00$, serum specimens were retested at a dilution of 1:400. $A \geqslant 0.15$ rise in net $O D$ of the postvaccination specimen over that of the prevaccination specimen was considered significant [17] (seroconversion).

\section{Statistical Methods}

Proportions were compared by $\chi^{2}$ or Fisher's exact test. GMTs were compared by $t$ tests using log-transformed data.

\section{Results}

\section{Safety}

CVD 103-HgR live oral cholera vaccine was well tolerated by the Thai adults. No diarrheal illness was observed in the open studies (studies 2 and 3), either in soldiers or in civilians. In the two placebo-controlled studies (studies 1 and 4), no increased rate of diarrheal episodes or other gastrointestinal adverse reactions was observed among vaccine than among placebo recipients. Diarrhea was recorded in $11 \%$ of 102 vaccinees compared with $12.5 \%$ of 104 controls in study 1 ; in study 4 diarrhea was observed in $2.5 \%$ of 119 vaccinees compared with $2.5 \%$ of 79 controls. One subject in the vaccine group in study 4 passed eight loose stools and sought health care. This event occurred 3 days after the subject had received his second $5 \times 10^{9} \mathrm{cfu}$ dose of vaccine. A coproculture was not obtained during the diarrheal episode. Notably, this subject did not develop a rise in vibriocidal antibody or in antitoxin levels, suggesting that the diarrheal episode was due to another cause.

\section{Immunogenicity}

Vibriocidal antibody response. In study 1 , only $20 \%$ of the 95 soldiers tested who received a single $5 \times 10^{8} \mathrm{cfu}$ dose of CVD $103-\mathrm{HgR}$ oral vaccine developed significant rises in vibriocidal antibody and only $2 \%$ reached titers $\geqslant 1: 2560$ (table 2). This serologic response was markedly inferior to that of adults in industrialized countries given this dose of vaccine and to that of the first 12 young Thai adults at Mahidol University who participated in the preliminary study of CVD $103-\mathrm{HgR}$ (92\% of whom had seroconversions in Inaba vibriocidal antibody with a peak GMT of 3417) [13]. Because of this unexpectedly poor serologic response, a second study was done in which 40 Thai soldiers were randomly allocated to receive a single $5 \times 10^{8} \mathrm{cfu}$ dose of CVD 103- $\mathrm{HgR}$, either from the same lot of vaccine that was used in study 1 or from another lot. Vibriocidal seroconversion was $25 \%$ in each group (table 2). In one of these groups, five subjects had vibriocidal titers $\geqslant 1: 2560$. Study 2 showed that the poor serologic response of Thai soldiers to a single $5 \times 10^{8} \mathrm{cfu}$ dose of
CVD 103-HgR was apparently a consistent finding and not due to one particular lot of vaccine. Moreover, the presence of moderate (and occasionally high) baseline titers of vibriocidal antibody in some soldiers suggested that within this population there exist individuals who are already immune to cholera, presumably by having had inapparent or clinically mild cholera previously. These observations also suggested that Thai soldiers might represent an inherently different host than Thai civilians, despite the fact that the baseline vibriocidal GMT of the latter also suggested some prior exposure to cholera in this endemic area.

To investigate these possibilities, study 3 was done in May 1989, when one lot of CVD 103-HgR was used to vaccinate Thai soldiers or civilians with a single $5 \times 10^{8}$ cfu dose. As summarized in table 2 , the identical lot of vaccine was significantly less immunogenic in soldiers than in civilians.

The observations made in study 3 led us to explore practical ways to enhance the serologic response of Thai soldiers to CVD 103-HgR. The design of study 4 permitted us to pursue several objectives. The first was a comparison of the immunogenicity of a single dose of CVD 103-HgR containing $5 \times$ $10^{9} \mathrm{cfu}$ rather than $5 \times 10^{8} \mathrm{cfu}$. The second objective was to assess the variability of the serologic response of several different groups of Thai soldiers to a single $5 \times 10^{9} \mathrm{cfu}$ dose of CVD $103-\mathrm{HgR}$. The last objective was to measure the relative immunogenicity of two $5 \times 10^{9} \mathrm{cfu}$ doses of CVD 103$\mathrm{HgR}$ given I week apart.

Table 3 summarizes the vibriocidal response in subjects in study 4 who received two $5 \times 10^{9} \mathrm{cfu}$ doses, one $5 \times 10^{9} \mathrm{cfu}$ dose, or one $5 \times 10^{8} \mathrm{cfu}$ dose of CVD 103-HgR vaccine. In this summary analysis that combines data from several different groups who received the same regimen, there appears to be a modest gradient of serologic response, with the two-dose $5 \times 10^{9} \mathrm{cfu}$ regimen stimulating the best response and the single $5 \times 10^{8} \mathrm{cfu}$ dose regimen eliciting the weakest response. The effect of giving a second dose of vaccine 1 week after the first $5 \times 10^{9} \mathrm{cfu}$ dose is also shown in table 3 . While 20 of 40 subjects seroconverted after one dose, the second dose led to only three additional seroconversions, raising the total number of seroconvertors to 23 of 40 .

Upon further analysis, the difference in GMT between the subjects who received two $5 \times 10^{9} \mathrm{cfu}$ doses of vaccine and those who received one $5 \times 10^{9} \mathrm{cfu}$ dose (table 3 ) is seen largely to be an artifact unrelated to the number of doses; that is, the marked difference in vibriocidal response among the various groups was already evident after just a single dose of oral vaccine. This is illustrated in table 4 , where the vibriocidal response of groups who received a single dose of $5 \times$ $10^{9} \mathrm{cfu}$ (B and C) can be compared with the vibriocidal response after the first $5 \times 10^{9} \mathrm{cfu}$ dose of vaccine of groups (A and F) who eventually received a second dose. For this reason, only baseline and 7-day postvaccination titers are compared. In this analysis the high GMT of group F has already occurred after just one oral dose of vaccine. Indeed, the 
Table 2. Serum vibriocidal and antitoxin responses of Thai soldiers and civilians after a single dose of $5 \times 10^{8} \mathrm{cfu}$ of CVD 103-HgR live oral cholera vaccine in field studies during 1988 and 1989 .

\begin{tabular}{|c|c|c|c|c|c|}
\hline \multirow{2}{*}{$\begin{array}{l}\text { Study, } \\
\text { group }(n)\end{array}$} & \multirow[b]{2}{*}{ \% seroconverting } & \multirow{2}{*}{$\begin{array}{c}\% \text { with titers } \\
\geqslant 1: 2560\end{array}$} & \multicolumn{2}{|c|}{$\begin{array}{l}\text { Geometric } \\
\text { mean titer }\end{array}$} & \multirow{2}{*}{$\begin{array}{l}\text { No. (\%) with } \\
\text { serum antitoxin } \\
\text { seroconversion }\end{array}$} \\
\hline & & & Day 0 & Peak & \\
\hline \multicolumn{6}{|l|}{1} \\
\hline Soldiers $(95)^{*}$ & 20 & 2 & 81 & 149 & $22 *(22)$ \\
\hline \multicolumn{6}{|l|}{2} \\
\hline Soldiers $^{\dagger}(20)$ & 25 & 25 & 219 & 557 & $5(25)$ \\
\hline Soldiers $^{\ddagger}(20)$ & 25 & 0 & 104 & 260 & $5(25)$ \\
\hline \multicolumn{6}{|l|}{3} \\
\hline Soldiers (33) & $39^{8}$ & $0^{\prime}$ & 80 & 238 & $3(27)^{n}$ \\
\hline Civilians (30) & $63^{8}$ & $40^{\prime}$ & 142 & 1470 & $16(53)^{\pi}$ \\
\hline
\end{tabular}

* Of 102 subjects vaccinated, paired sera were obtained from 95 .

${ }^{\dagger}$ Same vaccine lot as used in study 1.

$\ddagger$ Different lot from that used in study 1 .

${ }^{s} P=.079 .{ }^{I} P<.001$, two-tailed Fisher's exact test: ${ }^{1} P=.064, \chi^{2}$ test with Yates's correction.

GMT of group F on day 7 (597) after just one dose of vaccine is already significantly higher than that of group A (269), the group with the lowest GMT of the four groups. Comparing GMTs of all groups on day 7 (table 4 ) shows that a single $5 \times$ $10^{9} \mathrm{cfu}$ dose of CVD $103-\mathrm{HgR}$ always resulted in a higher GMT than did a single $5 \times 10^{8} \mathrm{cfu}$ dose of oral vaccine. The GMT (368) on day 7 after vaccination of the 80 subjects who had one $5 \times 10^{9}$ cfu dose of oral vaccine (includes the response after the first dose of the 40 vaccinees who went on to receive a second dose on day 7) was significantly higher than that (191) of the 39 subjects who received a single $5 \times 10^{8}$ cfu dose of vaccine $(P=.016)$.

The kinetics of the vibriocidal antibody responses measured on days 0,7 , and 21 in the various groups of soldiers who received the different immunization regimens is shown in figure 1 .

The high baseline vibriocidal titers recorded in many of the Thai soldiers suggested that previous antigenic experience with $V$. cholerae $\mathrm{O} 1$ rendered them already immune. In such subjects the lack of rise in serum vibriocidal antibodies might not adequately reflect the immunologic status of these individuals. To investigate this hypothesis, we analyzed the baseline vibriocidal antibody titers of seroconvertors and nonseroconvertors to determine if those of the latter were higher, thereby providing serologic evidence of prior antigenic contact with cholera vibrios. Among soldiers who received the $5 \times 10^{9} \mathrm{cfu}$ dose of vaccine, the baseline GMT of the 40 nonseroconvertors (132) was significantly higher than that of the seroconvertors $(45, P<.001)$. This inverse relationship also held true for the soldiers who received the $5 \times$ $10^{8} \mathrm{cfu}$ dose of vaccine. Among the 207 soldiers who received this dose, the prevaccination vibriocidal GMT of the 144 nonseroconvertors (112) was significantly higher than the baseline GMT of the 63 soldiers who seroconverted $(61$, $P<.001$ ). Seroconversion in relation to specific prevaccination Inaba vibriocidal titers is shown in table 5 for soldiers given either dose of vaccine. It is clear that the higher the baseline titer, the lower the percentage of seroconversion.

Among the 30 civilians who received a single $5 \times 10^{8} \mathrm{cfu}$ dose of vaccine, the baseline Inaba vibriocidal antibody level

Table 3. Serum vibriocidal antibody response of groups of volunteers after ingesting live oral cholera vaccine CVD 103-HgR (study 4, June 1991).

\begin{tabular}{lccccc}
\hline & & \multicolumn{3}{c}{$\begin{array}{c}\text { Geometric mean titer on } \\
\text { day }\end{array}$} \\
\cline { 4 - 6 } Vaccine regimen $(n)$ & \% seroconverting & $\begin{array}{c}\text { \% with postvaccine } \\
\text { titers } \geqslant 1: 2560\end{array}$ & 0 & 7 & 21 \\
\hline $5 \times 10^{9 *}, 2$ doses $s^{\dagger}(40)$ & 58 & 23 & 67 & 401 & 320 \\
$5 \times 10^{9}, 1$ dose $(40)$ & 43 & 20 & 100 & 337 & 220 \\
$5 \times 10^{8 *}, 1$ dose $(39)$ & 33 & 21 & 81 & 191 & 175 \\
Placebo, 1 dose $(39)$ & 2 & 0 & 87 & 101 & \\
\hline
\end{tabular}

* Colony-forming units in one dose of vaccine.

+ Doses given 7 days apart.

$\ddagger$ Seroconversion after one dose was $20(50 \%)$ of $40 ; 13 \%$ reached titers $\geqslant 1: 2560$. 
Table 4. Serum vibriocidal antibody response in groups of volunteers 7 days after ingesting a single dose of live oral cholera vaccine CVD $103-\mathrm{HgR}$.

\begin{tabular}{|c|c|c|c|c|}
\hline \multirow[b]{2}{*}{ Group $(n)$, regimen } & \multirow{2}{*}{$\begin{array}{c}\%(\mathrm{CI}) \text { with } \\
\text { vibriocidal } \\
\text { seroconversion }\end{array}$} & \multirow{2}{*}{$\begin{array}{c}\text { No. with } \\
\text { titers } \geqslant 1: 2560\end{array}$} & \multicolumn{2}{|c|}{ Geometric mean titer $(\mathrm{CI})$ on day } \\
\hline & & & 0 & 7 \\
\hline \multicolumn{5}{|l|}{$A(20)$} \\
\hline $5 \times 10^{9}$ & $50(27-73)$ & 4 & $51(31-85)$ & $269(154-469)$ \\
\hline \multicolumn{5}{|l|}{$\mathrm{B}(20)$} \\
\hline $5 \times 10^{9}$ & $40(23-64)$ & 2 & $121(63-235)$ & $279(165-470)$ \\
\hline \multicolumn{5}{|l|}{$C(20)$} \\
\hline $5 \times 10^{9}$ & $45(23-65)$ & 6 & $83(46-148)$ & $408(162-1029)$ \\
\hline \multicolumn{5}{|l|}{$F(20)$} \\
\hline $5 \times 10^{9}$ & $50(27-73)$ & 5 & $89(47-167)$ & $597(327-1092)$ \\
\hline \multicolumn{5}{|l|}{$D(19)$} \\
\hline $5 \times 10^{8}$ & $26(9-51)$ & 3 & $77(52-115)$ & $185(103-332)$ \\
\hline \multicolumn{5}{|l|}{$E(20)$} \\
\hline $5 \times 10^{8}$ & $40(19-64)$ & 2 & $86(53-139)$ & $197(111-351)$ \\
\hline \multicolumn{5}{|l|}{ All recipients $(80)$} \\
\hline $5 \times 10^{9}$ & 48 & 15 & 82 & $368^{*}$ \\
\hline \multicolumn{5}{|l|}{ All recipients (39) } \\
\hline $5 \times 10^{8}$ & 33 & 5 & 81 & $191^{*}$ \\
\hline
\end{tabular}

NOTE. All groups received one dose at the number of colony-forming units indicated. CI. $95 \%$ confidence interval.

${ }^{*} P=.016$

among the 11 nonseroconvertors (GMT $=265)$ was significantly higher than that of the 19 seroconvertors (GMT = $100 ; P=.02, t$ test $)$. The percentage of seroconversion in the civilians also diminished with increasing baseline titer (table 5 ). However, among the civilians given a single dose of $5 \times$ $10^{8} \mathrm{cfu}$, significantly higher percentages of seroconversion were observed among those who had baseline titers of $\leqslant 1: 40$; $6(100 \%)$ of 6 seroconverted. In contrast, of the 64 soldiers with baseline titers $\leqslant 1: 40$ who received a single $5 \times 10^{8} \mathrm{cfu}$ dose of oral vaccine, only $45 \%$ seroconverted $(P=.025)$. The percentage of seroconversion in soldiers with low $(\leqslant 1: 40)$ baseline titers was significantly higher when they were given a log-higher dose of vaccine ( $79 \%$ vs. $45 \%, P=.006$ ).

\section{Antitoxin Response}

The percentages of IgG antitoxin seroconversion detected in serum generally paralleled the patterns of vibriocidal seroconversion in the four studies. Data from studies $1-3$ are summarized in table 2 . In study 4 , significant rises in serum IgG antitoxin occurred in $58 \%$ of the 40 soldiers who received two $5 \times 10^{9}$ cfu doses, $43 \%$ of the 40 who got one $5 \times$ $10^{9} \mathrm{cfu}$ dose, and $41 \%$ of the 39 soldiers who got a single $5 \times$ $10^{8} \mathrm{cfu}$ dose of vaccine.

\section{Discussion}

The critical role played by antibacterial, rather than antitoxic, immunity in long-term protection against cholera is becoming increasingly recognized $[4,14,15,18]$. Hereto- fore, evaluations of the immunogenicity of CVD $103-\mathrm{HgR}$ in various populations have used serum vibriocidal antibody response as the measure of antibacterial immunity and as a correlate of elicited protection [6, 12, 13]. In populations from industrialized countries, representing individuals who lack background immunity to $V$. cholerae $\mathrm{O} 1$, this measurement of serum antibody has been remarkably useful for determining vaccine "take."

While a single $5 \times 10^{8} \mathrm{cfu}$ dose of CVD 103-HgR has consistently elicited $80 \%-95 \%$ vibriocidal seroconversion in persons from industrialized countries, it has been equally consistent in eliciting only $20 \%-50 \%$ seroconversion in Thai soldiers. The series of studies in Thailand provides several insights into the reasons for this. One observation is that the vibriocidal response of soldiers to a $5 \times 10^{8} \mathrm{cfu}$ dose of CVD $103-\mathrm{HgR}$ is significantly less than that of civilians of similar age, demonstrating host differences (table 2). Civilians were more often urban and from middle socioeconomic background, while the soldiers were more commonly of rural and lower socioeconomic background.

We hypothesize that at least two factors play a role in the lower serum vibriocidal antibody seroconversion in soldiers: Preexisting background immunity against $V$. cholerae $\mathrm{Ol}$ and levels of microflora in the proximal small intestine. Background immunity in some soldiers would limit intestinal colonization of the vaccine strain in these individuals. While live oral cholera vaccine would likely boost mucosal immunity in the intestine, such local boosting might not be reflected by a rise in serum vibriocidal antibodies. Indeed, three observations strongly support this contention. 


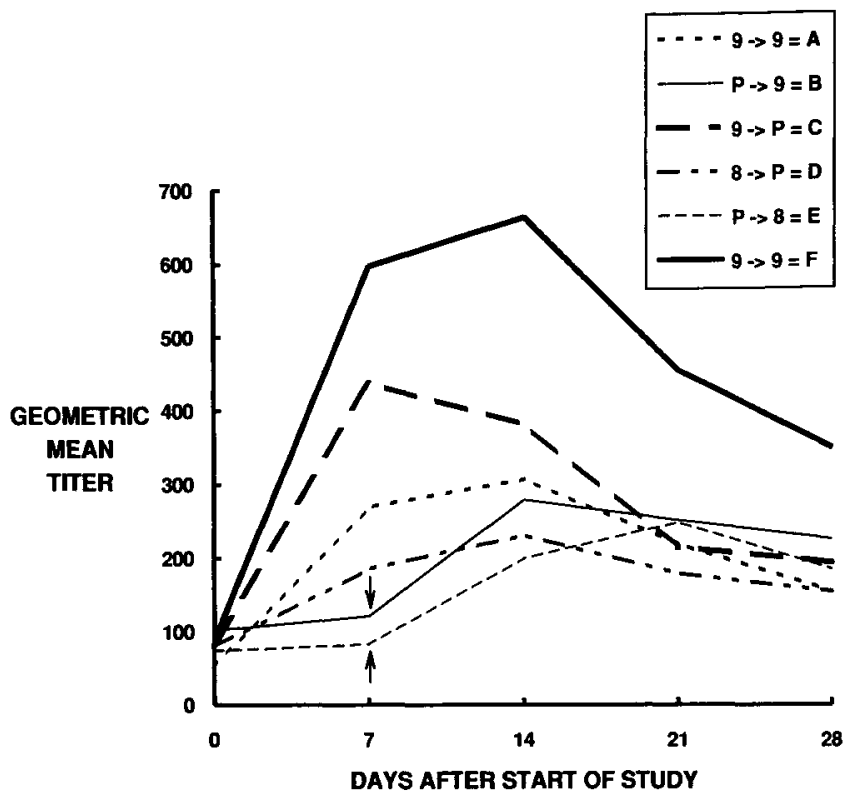

Figure 1. Kinetics of serum vibriocidal antibody response in crossover study of live oral cholera vaccine strain CVD 103-HgR (study 4). Volunteers received either placebo (killed Escherichia coli $\mathrm{K} 12$ ) or vaccine on day 0 or 7 . Groups $A$ and $F$ received $5 \times$ $10^{9} \mathrm{cfu}$ of vaccine on both days. Groups $B$ and $E$ received placebo on day 0 and $5 \times 10^{9}$ cfu and $5 \times 10^{8} \mathrm{cfu}$, respectively, on day 7 (arrows). Groups C and D received $5 \times 10^{9}$ and $5 \times 10^{8}$, respectively, on day 0 , and both received placebo on day 7 .

First, some soldiers do have quite elevated vibriocidal titers at baseline. A vibriocidal antibody titer of 1:1280 has been cited as suggestive of recent cholera infection [10]. A total of $9(2.4 \%)$ of the 368 soldiers who participated in these studies whose sera were tested (284 vaccinees and 84 controls) had titers $\geqslant 1: 1280$ before vaccination despite the lack of previous cholera vaccination. Second, seroconversion after CVD $103-\mathrm{HgR}$ vaccination was found to be strongly inversely related to the baseline vibriocidal antibody titer. Nonseroconvertors had a significantly higher baseline GMT than did seroconvertors, and the higher the baseline titer, the lower the percentage of seroconversion (table 5). The final evidence comes from groups of North American volunteers who developed experimental cholera while serving as controls in vaccine efficacy studies and who were then rechallenged with pathogenic $V$. cholerae $\mathrm{O} 12$ months to 3 years later [10, 18-20]. These volunteers showed prominent rises in titer of serum vibriocidal antibody after their initial clinical cholera infection, which fell over 1-12 months to a level that was nevertheless above baseline [10]. While these volunteers were solidly protected against cholera upon rechallenge with an $\mathrm{ID}_{90}$ of wild-type vibrios $[10,18-20]$, they either did not show rises in serum vibriocidal antibody upon rechallenge or showed only modest rises $[10,20]$.

The above observations demonstrate that while serum vibriocidal antibody is an excellent means to document the successful stimulation of antibacterial immunity in individuals who lack previous antigenic stimulation by $V$. cholerae $\mathrm{Ol}$ (such as subjects in industrialized countries), it is not a sensitive measure for determining whether successful boosting has occurred in antigenically primed individuals (such as adults in areas endemic for cholera). Future immunogenicity studies will need to take this into account and may have to include measurements of fecal or jejunal secretory IgA antibodies and gut-derived trafficking IgA antibody-secreting cells (detected in peripheral blood) to ascertain whether intestinal mucosal immunity has been successfully boosted in vaccinees with antecedent immunity.

Another factor affecting the take after administration of live oral bacterial vaccines may be differences in intestinal microflora. Adults and children living under low socioeconomic conditions in less-developed countries are known to have increased levels of anerobic and coliform microflora in their proximal small intestine compared with persons from industrialized countries [21-23]. These elevated levels of proximal intestinal microflora are associated with morphologic changes that include flattening of the villi $[24,25]$. These microflora may serve as a barrier to successful vaccine take by CVD $103-\mathrm{HgR}$, which must compete with existing microflora to colonize and elicit immune responses. Some of the Thai civilians also had elevated baseline vibriocidal titers and, as expected, showed less seroconversion (table 5). However, civilians with low baseline titers had significantly higher percentages of seroconversion than did soldiers with the same baseline titers. This result would suggest that in the soldiers other factors, in addition to baseline titer, were operative in diminishing the vaccine take. We hypothesize that the

Table 5. Preimmunization Inaba vibriocidal antibody titers and numbers of Thai civilians and soldiers who seroconverted after ingesting a single dose of CVD-103Hg-R live oral cholera vaccine.

\begin{tabular}{lccc}
\hline & \multicolumn{3}{c}{ Group, cfu } \\
\cline { 2 - 4 } & & \multicolumn{2}{c}{ Soldiers } \\
\cline { 2 - 4 } Titer & Civilians & $5 \times 10^{9}$ & $5 \times 10^{8}$ \\
\hline $1: 10$ & $5 \times 10^{8}$ & $8 / 8(100)$ & $9 / 16(56)$ \\
$1: 20$ & $3 / 3(100)$ & $8 / 11(73)$ & $3 / 13(23)$ \\
$1: 40$ & $2 / 2(100)$ & $6 / 9(67)$ & $17 / 35(49)$ \\
$1: 80$ & $1 / 1(100)$ & $8 / 21(38)$ & $17 / 51(33)$ \\
$1: 160$ & $3 / 3(100)$ & $6 / 17(35)$ & $11 / 51(22)$ \\
$1: 320$ & $3 / 9(33)$ & $3 / 9(33)$ & $3 / 26(12)$ \\
$1: 640$ & $4 / 7(57)$ & $0 / 2(0)$ & $3 / 10(30)$ \\
$\geqslant 1: 1280$ & $3 / 4(75)$ & $0 / 1(0)$ & $0 / 5(0)$ \\
$\leqslant 1: 40$ & $0 / 1(0)$ & $22 / 28(79)$ & $29 / 64(45)$ \\
$>1: 40$ & $6 / 6^{*+}(100)$ & $17 / 50(34)$ & $34 / 143(24)$ \\
\hline
\end{tabular}

NOTE. Data are given as no. of vaccinees with fourfold or greater rises/ no. vaccinated $(\%)$. cfu. colony-forming units.

* $6 / 6$ vs. $29 / 64, P=.025 ;{ }^{\dagger} 22 / 28$ vs. $29 / 64, P=.006$; two-tailed Fisher's exact test. 
combination of elevated baseline titers and increased levels of microflora in the proximal small intestine (if present) in the soldiers might work in tandem to effectively diminish the dose of CVD 103-HgR organisms that reach the intestinal immune system.

On the basis of the above observations, we asked whether simply increasing the dose of CVD 103-HgR to $5 \times 10^{9} \mathrm{cfu}$ could adequately enhance immunogenicity. In the fourth of our studies of Thai adults reported here, a single dose of CVD 103-HgR containing $5 \times 10^{9} \mathrm{cfu}$ elicited higher GMTs than did a single dose containing $5 \times 10^{8} \mathrm{cfu}$. More dramatic supportive data come from studies in Indonesian children 5-9 years old, in whom a single $5 \times 10^{8}$ cfu dose of CVD $103-\mathrm{HgR}$ stimulated seroconversion in only $16 \%$, while a single $5 \times 10^{9} \mathrm{cfu}$ dose of the same lot of vaccine elicited seroconversion in $79 \%$ (unpublished data).

This series of studies emphasizes the complexity of the oral route of immunization, the importance of obtaining data from several immunogenicity studies before drawing conclusions, and the need to develop simple, practical ways of measuring boosting of the intestinal immune system in individuals with background immunity. In these various studies of immunogenicity we measure serologic responses as a proxy for the elicitation of protective immunity. Ultimately, direct evidence of protection in these populations must be garnered from carefully designed and conducted field trials of efficacy under natural conditions of challenge. The results of such studies will put the serologic responses in proper perspective.

\section{References}

1. World Health Organization, Diarrhoeal Diseases Control Programme. Biomedical and epidemiological research priorities of global scientific working groups. 1987 Geneva: WHO, 1987; WHO/CDD86.8. Rev 1.

2. Levine MM. Vaccines against enteric infections. Lancet 1990;335: 958-61.

3. Glass RI, Claeson M, Blake PA, Waldman RJ, Pierce NF. Cholera in Africa: lessons on transmission and control for Latin America. Lancet 1991;338:791-5.

4. Clemens JD, Sack DA, Harris JR, et al. Field trial of oral cholera vaccines in Bangladesh: results from three-year follow-up. Lancet 1990;335:270-3.

5. Kaper JB, Lockman H, Baldini MM, Levine MM. Recombinant live oral cholera vaccine. Biotechnology 1984;2:345-9.

6. Levine MM, Kaper JB, Herrington D, et al. Safety, immunogenicity and efficacy of recombinant live oral cholera vaccine CVD 103 and CVD 103-HgR. Lancet 1988;2:467-70.

7. Kaper JB, Levine MM. Recombinant attenuated Vibrio cholerae strains used as live oral vaccines. Res Microbiol 1990;141:901-6.

8. Mosley WH, Beneson AS, Barui R. A serological survey for cholera antibodies in rural east Pakistan. I. The distribution of antibody in the control population of a cholera vaccine field-trial area and the relation of antibody titer to the pattern of endemic cholera. Bull WHO 1968;38:327-34

9. Mosley WH, Aziz AKM, Rahman ASMM, Chowdhury AKMA. Ahmed A. Field trials of monovalent Ogawa and Inaba cholera vaccines in rural Bangladesh-three years of observation. Bull WHO 1973;49:381-7.

10. Clemens JD, Stanton BF, Chakraborty J, et al. B subunit-whole cell and whole cell-only oral vaccines against cholera: studies on reactogenicity and immunogenicity. J Infect Dis 1987;155:79-85.

11. Levine MM, Pierce NF. Immunity and vaccine development. In: Greenough WB III, Barua D, eds. Cholera. New York: Plenum Press, 1991.

12. Cryz SC Jr, Levine MM, Kaper JB, Furer E, Althaus B. Randomized double-blind placebo controlled trial to evaluate the safety and immunogenicity of the live oral cholera vaccine strain CVD 103-HgR in Swiss adults. Vaccine 1990;8:577-80.

13. Migasena S, Pitisuttitham P, Prayurahong B, et al. Preliminary assessment of the safety and immunogenicity of live oral cholera vaccine strain CVD 103- $\mathrm{HgR}$ in healthy Thai adults. Infect Immun 1989;57:3261-4.

14. Levine MM, Black RE, Clements ML, et al. Evaluation in man of attenuated Vibrio cholerae El Tor Ogawa strain Texas Star-SR as a live oral vaccine. Infect Immun 1984;43:515-22.

15. Levine MM, Kaper KB, Herrington DA, et al. Volunteer studies of deletion mutants of Vibrio cholerae $\mathrm{OI}$ prepared by recombinant techniques. Infect Immun 1988;56:161-7.

16. Benenson AS, Saad A, Mosley WH. Serological studies in cholera. 2. The vibriocidal antibody response of cholera patients determined by a microtechnique. Bull WHO 1968;38:277-85.

17. Levine MM, Young CR, Black RE, Takeda Y, Finkelstein RA. Enzyme-linked immunosorbent assay to measure antibodies to purified heat-labile enterotoxins from human and porcine strains of Escherichia coli and to cholera toxin: application in serodiagnosis and seroepidemiology. J Clin Microbiol 1985;21:174-9.

18. Levine MM. Nalin DR, Craig JP, et al. Immunity to cholera in man: relative role of antibacterial versus antitoxic immunity, Trans $\mathrm{R}$ Soc Trop Med Hyg 1979;73:3-9.

19. Levine MM. Immunity to cholera as evaluated in volunteers. In: Ouchterlony O, Holmgren J, eds. Cholera and related diarrhoeas: molecular aspects of a global health problem. Basel: S Karger, 1980:195203.

20. Levine MM, Black RE, Clements ML, Cisneros L, Nalin DR, Young $\mathrm{CR}$. The quality and duration of infection-derived immunity to cholera. $J$ Infect Dis $1981 ; 143: 818-20$.

21. Mata LJ, Jimenez F, Cordon M, et al. Gastrointestinal flora of children with protein calorie malnutrition. Am J Clin Nutr 1972;25:118-26.

22. Gracey M, Suharyono, Sunon, Stone DE. Microbial contamination of the gut: another feature of malnutrition. Am J Clin Nutr 1973;26:1170-4.

23. Bhat P. Shantakumari S, Rajan D, et al. Gastroenterology 1972;62:1121 .

24. Lindenbaum J, Alam AKMJ, Kent TH. Subclinical small intestinal disease in east Pakistan. BMJ 1966;2:1616-9.

25. Sprinz H, Srbhibhadh R, Gangarosa EJ, Benyajati C, Kundel D. Halstead S. Biopsy of small bowel of Thai people. Am J Clin Pathol $1962 ; 38: 43-51$ 